\title{
Controllable manipulation and entanglement of macroscopic quantum states in coupled charge qubits
}

\author{
J. Q. You, ${ }^{1}$ J. S. Tsai, ${ }^{1,2}$ and Franco Nori ${ }^{1,3, *}$ \\ ${ }^{1}$ Frontier Research System, The Institute of Physical and Chemical Research (RIKEN), Wako-shi 351-0198, Japan \\ ${ }^{2}$ NEC Fundamental Research Laboratories, Tsukuba, Ibaraki 305-8051, Japan \\ ${ }^{3}$ Center for Theoretical Physics, Physics Department, Center for the Study of Complex Systems, The University of Michigan, \\ Ann Arbor, Michigan 48109-1120, USA
}

(Received 25 February 2003; published 22 July 2003)

\begin{abstract}
We present an experimentally implementable method to couple Josephson charge qubits and to generate and detect macroscopic entangled states. A large-junction superconducting quantum interference device is used in the qubit circuit for both coupling qubits and implementing the readout. Also, we explicitly show how to achieve a microwave-assisted macroscopic entanglement in the coupled-qubit system.
\end{abstract}

DOI: 10.1103/PhysRevB.68.024510

PACS number(s): 85.25.-j, 03.65.Ud, 03.67.Lx

\section{INTRODUCTION}

Quantum-mechanical systems can exploit the fundamental properties of superposition and entanglement to process information in an efficient and powerful way that no classical device can do. Recently, Josephson-junction circuits have received renewed attention because these may be used as qubits in a quantum computer. ${ }^{1}$ Based on the charge and phase degrees of freedom in Josephson-junction devices, charge $e^{2,3}$ and phase qubits ${ }^{4-6}$ have been developed. Also, a type of solid-state qubit can be realized in a large-area current-biased Josephson junction. ${ }^{7,8}$

Experimentally, coherent oscillations were demonstrated in a Josephson charge qubit prepared in a superposition of two charge states. ${ }^{2}$ More recent experimental measurements ${ }^{9}$ showed that the charge qubit at suitable working points can have a sufficiently high quality of coherence $\left(Q_{\varphi} \approx 2.5\right.$ $\times 10^{4}$ ), corresponding to a decoherence time $T_{\varphi} \approx 500 \mathrm{~ns}$. Current-biased Josephson junctions can also have long decoherence times ${ }^{7,8}$ and $Q_{\varphi}$ can reach $10^{4}$. These exciting experimental advancements demonstrate the potential of Josephson qubits for manufacturing macroscopic quantummechanical machines. Towards the practical implementation of a solid-state quantum computer, the next important step would be the coupling of two qubits and then scaling up the architecture to many qubits.

In this work, we present an experimentally implementable method to couple two Josephson charge qubits and to generate and detect macroscopic quantum entangled states in this charge-qubit system. Motivated by very recent experimental results, ${ }^{9}$ we employ a superconducting quantum interference device (SQUID) with two large Josephson junctions to implement the readout. The generation of the macroscopic entanglement is assisted by applying a microwave field to each charge qubit. The key advantage of our design is that the SQUID can also produce an experimentally feasible and controllable coupling between the two charge qubits. As verified in a single qubit, ${ }^{9}$ the coupled charge qubits may be well decoupled from the readout system when the measurement is not implemented. Moreover, our design can be readily extended to coupled multiple ${ }^{10}$ qubits as well as any selected pairs (not necessarily neighbors).

The paper is organized as follows. In Sec. II, the controllable coupling between two charge qubits is proposed using a large Josephson junction or a large-junction dc SQUID. Also, we demonstrate how this interbit coupling can be conveniently used to generate the controlled-phase-shift gate. In Sec. III, we study the microwave-assisted macroscopic quantum entanglement in the coupled charge qubits, where the microwave fields are coupled to the qubits via gate capacitances. Section IV focuses on the readout of the quantum states in the coupled-qubit system. Finally, the discussion and conclusion are given in Sec. V.

\section{A. Other qubit coupling schemes}

A different type of interbit coupling from the one studied here was proposed using the Coulomb interaction between charges on the islands of the charge qubits. ${ }^{11}$ As pointed out in Ref. 1, the interbit coupling in this scheme is not switchable and also it is hard to make the system scalable because only neighboring qubits can be coupled. Implementations of quantum algorithms such as the Deutsch and BernsteinVazirani algorithms were studied using a system of Josephson charge qubits, ${ }^{12}$ where it was proposed that the nearestneighbor superconducting islands would be coupled by tunable dc SQUIDs. In Ref. 13, a pair of charge qubits were proposed to be capacitively coupled to a current-biased Josephson junction where, by varying the bias current, the junction can be tuned in and out of resonance with the qubits coupled to it.

Another different type of interbit coupling was proposed $^{1,3}$ in terms of the oscillator modes in an $L C$ circuit. In contrast, we use a large junction or a large-junction dc SQUID (but no $L C$ circuit) to couple the charge qubits. In our scheme, both $\mathrm{dc}$ and ac supercurrents can flow through the charge-qubit circuit, while in Refs. 1 and 3 only ac supercurrents can flow through the circuit. These yield different interbit couplings (e.g., the $\sigma_{y} \sigma_{y}$ type $\mathrm{e}^{1,3}$ as opposed to $\sigma_{x} \sigma_{x}$ in our proposal). As revealed in Ref. 10, the $\sigma_{x} \sigma_{x}$ type interbit coupling can be conveniently used to formulate an efficient quantum computing scheme. 


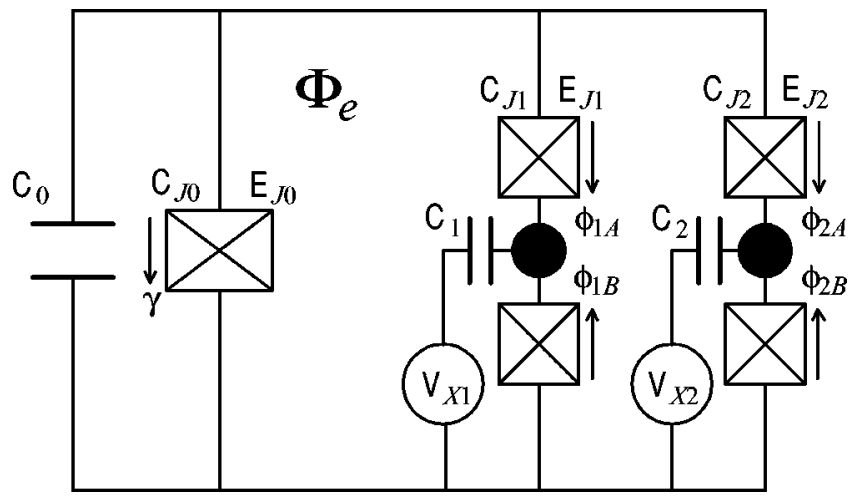

FIG. 1. Schematic diagram of two charge qubits coupled by a large Josephson junction (denoted by a square with an $\mathrm{X}$ inside) of coupling energy $E_{J 0}$ and capacitance $C_{J 0}$. To make the effective charging energy of the large Josephson junction as small as required, a large capacitance $C_{0}$ is placed close to and in parallel with it. Each filled circle denotes a superconducting island, the Cooperpair box, which is biased by a voltage $V_{X i}$ via the gate capacitance $C_{i}$ and coupled to the bulk superconductors by two identical small Josephson junctions (each with a coupling energy $E_{J i}$ and a capacitance $C_{J i}$ ). Here the arrow near each Josephson junction denotes the chosen direction for the positive phase drop across the corresponding junction.

Moreover, the calculated interbit-coupling terms in Refs. 1 and 3 only apply to the case in which the following two conditions are met:

(i) The eigenfrequency $\omega_{L C}$ of the $L C$ circuit is much faster than the quantum manipulation frequencies. This condition limits the allowed number $N$ of the qubits in the circuit because $\omega_{L C}$ scales with $1 / \sqrt{N}$. In other words, this implies that the circuits in Refs. 1 and 3 are not really scalable.

(ii) The phase conjugate to the total charge on the qubit capacitors fluctuates weakly. Our interbit-coupling approach discussed below is free from these two limitations.

\section{CONTROLLABLE COUPLING OF CHARGE QUBITS}

\section{A. Coupling qubits with a large junction}

We first use a large Josephson junction to couple two charge qubits (see Fig. 1). Each qubit is realized by a Cooper-pair box, where a superconducting island with excess charge $\hat{Q}_{i}=2 e \hat{n}_{i}(i=1,2)$ is weakly coupled to the bulk superconductors via two identical small junctions (with Josephson coupling energy $E_{J i}$ and capacitance $C_{J i}$ ) and biased by an applied voltage $V_{X i}$ through a gate capacitance $C_{i}$. The large Josephson junction on the left has a coupling energy $E_{J 0}$ (much larger than $E_{J i}$ ) and a capacitance $C_{J 0}$. As in the single-qubit case, ${ }^{9}$ close to the large Josephson junction, we also place a large capacitance $C_{0}$ in parallel with it, so that the effective charging energy of the large Josephson junction can be ignored (even though the capacitance of the large junction might not be large enough). Moreover, we assume that the inductance of the qubit circuit (i.e., the two Cooperpair boxes with the nearby junctions, and the superconducting lines connecting these two qubits with the large Joseph- son junction) is much smaller than the Josephson inductance of the large junction. The Hamiltonian of the system can be written as

$$
\begin{aligned}
H= & \sum_{i=1}^{2}\left[E_{c i}\left(\hat{n}_{i}-n_{X i}\right)^{2}-E_{J i}\left(\cos \hat{\phi}_{i A}+\cos \hat{\phi}_{i B}\right)\right] \\
& -E_{J 0} \cos \hat{\gamma}
\end{aligned}
$$

where

$$
E_{c i}=\frac{2 e^{2}}{C_{i}+2 C_{J i}}
$$

is the charging energy of the superconducting island and $n_{X i}=C_{i} V_{X i} / 2 e$ is the reduced offset charge (in units of $2 e$ ) induced by the gate voltage. Flux quantization around loops containing the phase drops of the involved junctions gives the constraint

$$
\hat{\phi}_{i A}-\hat{\phi}_{i B}-\hat{\gamma}+\frac{2 \pi \Phi_{e}}{\Phi_{0}}=0, \quad i=1,2,
$$

which gives

$$
\begin{gathered}
\hat{\phi}_{i A}=\hat{\phi}_{i}-\left(\frac{\pi \Phi_{e}}{\Phi_{0}}-\frac{1}{2} \hat{\gamma}\right), \\
\hat{\phi}_{i B}=\hat{\phi}_{i}+\left(\frac{\pi \Phi_{e}}{\Phi_{0}}-\frac{1}{2} \hat{\gamma}\right),
\end{gathered}
$$

where the average phase drop $\hat{\phi}_{i}=\left(\hat{\phi}_{i A}+\hat{\phi}_{i B}\right) / 2$ is canonically conjugate to the number, $\hat{n}_{i}$, of the excess Cooper pairs on the $i$ th superconducting island:

$$
\left[\hat{\phi}_{j}, \hat{n}_{j}\right]=i, \quad j=1,2 \text {. }
$$

Here $\hat{\phi}_{i A}$ and $\hat{\phi}_{i B}(i=1,2)$ are the phase drops across the small Josephson junctions above $(A)$ and below $(B)$ the $i$ th Cooper-pair box.

The Hamiltonian (1) can be rewritten as

$$
\begin{aligned}
H= & \sum_{i=1}^{2}\left[E_{c i}\left(\hat{n}_{i}-n_{X i}\right)^{2}-2 E_{J i} \cos \left(\frac{\pi \Phi_{e}}{\Phi_{0}}-\frac{1}{2} \hat{\gamma}\right) \cos \hat{\phi}\right] \\
& -E_{J 0} \cos \hat{\gamma} .
\end{aligned}
$$

The externally applied flux $\Phi_{e}$ threads the area between the large Josephson junction and the left Cooper-pair box. It induces circulating supercurrents in the qubit circuit. The total circulating supercurrent $\hat{I}$ has contributions from the two charge qubits:

$$
\hat{I}=\hat{I}_{1}+\hat{I}_{2}
$$

where

$$
\hat{I}_{i}=2 I_{c i} \sin \left(\frac{\pi \Phi_{e}}{\Phi_{0}}-\frac{1}{2} \hat{\gamma}\right) \cos \hat{\phi}_{i},
$$

with $I_{c i}=\pi E_{J i} / \Phi_{0}$. This total supercurrent flows through the large Josephson junction and it can also be written as

$$
\hat{I}=I_{0} \sin \hat{\gamma}
$$

with $I_{0}=2 \pi E_{J 0} / \Phi_{0}$. From Eqs. (6)-(8) it follows that 


$$
I_{0} \sin \hat{\gamma}=2 \sin \left(\frac{\pi \Phi_{e}}{\Phi_{0}}-\frac{1}{2} \hat{\gamma}\right)\left(I_{c 1} \cos \hat{\phi}_{1}+I_{c 2} \cos \hat{\phi}_{2}\right) .
$$

When the coupling energy $E_{J i}=\Phi_{0} I_{c i} / \pi$ of each Josephson junction connected to the charge box is much smaller than that of the large Josephson junction in the circuit, the phase drop $\hat{\gamma}$ across the large junction will be small. Expanding the operator functions of $\hat{\gamma}$ in Eq. (9) into a series and retaining the terms up to second order of the parameters

$$
\eta_{i}=\frac{I_{c i}}{I_{0}} \quad(<1), \quad i=1,2,
$$

we have

$$
\begin{aligned}
\hat{\gamma}= & 2 \sin \left(\frac{\pi \Phi_{e}}{\Phi_{0}}\right)\left(\eta_{1} \cos \hat{\phi}_{1}+\eta_{2} \cos \hat{\phi}_{2}\right) \\
& -\sin \left(\frac{2 \pi \Phi_{e}}{\Phi_{0}}\right)\left(\eta_{1} \cos \hat{\phi}_{1}+\eta_{2} \cos \hat{\phi}_{2}\right)^{2} .
\end{aligned}
$$

It is clear that the phase drop $\hat{\gamma}$ across the large Josephson junction is controllable via the applied flux $\Phi_{e}$.

For Hamiltonian (5), we also expand the operator functions of $\hat{\gamma}$ into a series and retain the terms up to second order of $\eta_{i}$. Moreover, we consider the charging regime with $E_{c i}$ much larger than $E_{J i}$. Also, we assume that the temperature is low enough $\left(k_{B} T \ll E_{c i}\right)$ and the superconducting gap is larger than $E_{c i}$, so that quasiparticle tunneling is strongly suppressed. In this case, only the lowest two charge states are important for each qubit operating around the degeneracy point $V_{X i}=\left(2 n_{i}+1\right) e / C_{i}$. In the spin- $\frac{1}{2}$ representation based on the charge states $\left|n_{i}\right\rangle \equiv|\uparrow\rangle_{i}$, and $\left|n_{i}+1\right\rangle \equiv|\downarrow\rangle_{i}$ of each Cooper-pair box, the Hamiltonian of the system can be reduced to

$$
H=\sum_{i=1}^{2}\left[\varepsilon_{i}\left(V_{X i}\right) \sigma_{z}^{(i)}-\bar{E}_{J i} \sigma_{x}^{(i)}\right]-\chi \sigma_{x}^{(1)} \sigma_{x}^{(2)},
$$

with

$$
\varepsilon_{i}\left(V_{X i}\right)=\frac{1}{2} E_{c i}\left[\frac{C_{i} V_{X i}}{e}-\left(2 n_{i}+1\right)\right]
$$

and

$$
\bar{E}_{J i}=E_{J i} \cos \left(\frac{\pi \Phi_{e}}{\Phi_{0}}\right) \xi_{i}
$$

where

$$
\xi_{i}=1-\frac{3}{8}\left(\eta_{i}^{2}+3 \eta_{j}^{2}\right) \sin ^{2}\left(\frac{\pi \Phi_{e}}{\Phi_{0}}\right),
$$

and $i, j=1,2(i \neq j)$. The interbit coupling $\chi$ is given by

$$
\chi=L_{J} I_{c 1} I_{c 2} \sin ^{2}\left(\frac{\pi \Phi_{e}}{\Phi_{0}}\right),
$$

where the large Josephson junction acts as an effective inductance of value

$$
L_{J}=\frac{\Phi_{0}}{2 \pi I_{0}} .
$$

It is clear that the interbit coupling is switched off at $\Phi_{e}$ $=0$. It is well known that a large Josephson junction can act as an inductance (e.g., Ref. 1). Here we explicitly show a specific way that it can be used to couple qubits.

Retaining up to second-order terms in the expansion parameters $\eta_{i}$, the total circulating current $\hat{I}$ can be written as

$$
\begin{aligned}
\hat{I}= & 2 \sin \left(\frac{\pi \Phi_{e}}{\Phi_{0}}\right)\left(I_{c 1} \cos \hat{\phi}_{1}+I_{c 2} \cos \hat{\phi}_{2}\right) \\
& -\frac{1}{I_{0}} \sin \left(\frac{2 \pi \Phi_{e}}{\Phi_{0}}\right)\left(I_{c 1} \cos \hat{\phi}_{1}+I_{c 2} \cos \hat{\phi}_{2}\right)^{2} .
\end{aligned}
$$

In the spin- $-\frac{1}{2}$ representation, it is given by

$$
\begin{aligned}
\hat{I}= & \sin \left(\frac{\pi \Phi_{e}}{\Phi_{0}}\right)\left[I_{c 1} \sigma_{x}^{(1)}+I_{c 2} \sigma_{x}^{(2)}\right] \\
& -\frac{1}{4 I_{0}} \sin \left(\frac{2 \pi \Phi_{e}}{\Phi_{0}}\right)\left[I_{c 1}^{2}+I_{c 2}^{2}+2 I_{c 1} I_{c 2} \sigma_{x}^{(1)} \sigma_{x}^{(2)}\right],
\end{aligned}
$$

which depends on the states of the charge-qubit system.

\section{B. Coupling qubits with a SQUID}

There are somewhat conflicting requirements imposed on this circuit. To obtain a large value for the effective Josephson inductance $L_{J}=\Phi_{0} / 2 \pi I_{0}$, a relatively small $I_{0}$ is needed, so that a large interbit coupling can be achieved. However, when the large Josephson junction is also employed for a readout, it is desirable to use a large $I_{0}$. This permits a larger range of $I_{b}$, so that a higher resolution in distinguishing qubit states can be achieved in the quantum measurement based on the switching of the supercurrent through the large junction.

These two opposite requirements can be conveniently solved if the leftmost large Josephson junction in Fig. 1 is replaced by a symmetric dc SQUID with two sufficiently large junctions (see Fig. 2). Instead of $\Phi_{e}$ inside the circuit loop between $E_{J 0}$ and the first qubit (as in Fig. 1), we now apply a flux $\Phi_{s}$ inside the large-junction dc SQUID loop (see Fig. 2). This SQUID can be used both for coupling the two charge qubits and implementing the readout. When the readout is not active $\left(I_{b}=0\right)$, we can choose a suitable flux $\Phi_{s}$ inside the SQUID loop to generate a larger interbit coupling. For $I_{b}=0$, the reduced Hamiltonian of the coupled-qubit system and the total circulating current $I$ have the same forms as in Eqs. (12) and (19), but with $\Phi_{e}$ and $I_{0}$ replaced by $\frac{1}{2} \Phi_{s}$ and

$$
I_{0}=2 I_{0}^{s} \cos \left(\frac{\pi \Phi_{s}}{\Phi_{0}}\right)
$$

where $I_{0}^{s}=2 \pi E_{J 0}^{s} / \Phi_{0}$. When the readout is active (see Sec. IV), $\Phi_{s}$ is chosen as zero to obtain a larger effective Josephson coupling energy. 


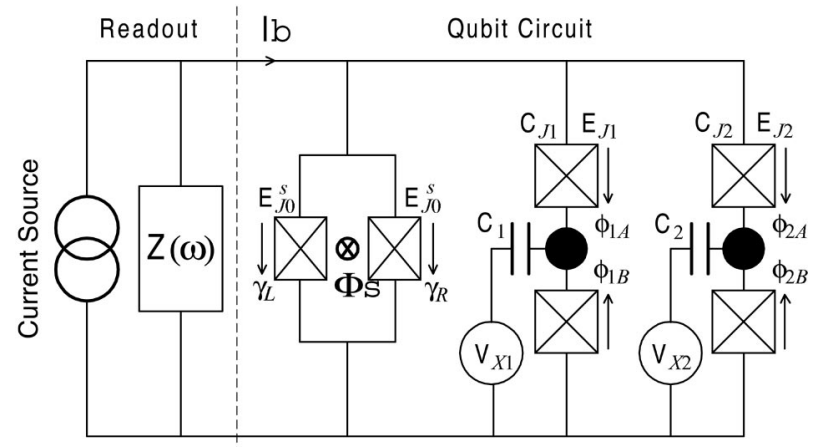

FIG. 2. Schematic diagram of the coupled-qubit circuit with a biased-current source of impedance $Z(\omega)$. The dc SQUID, with two junctions of large $E_{J 0}^{s}$, plays the role of both coupling the charge qubits and implementing the readout. Here the large capacitance $C_{0}$ placed close to and in parallel with the dc SQUID is included in the impedance $Z(\omega)$.

\section{Controlled-phase-shift gate}

When the system works at the degeneracy points with $\varepsilon_{i}\left(V_{X i}\right)=0$, the Hamiltonian becomes

$$
H=-\bar{E}_{J 1} \sigma_{x}^{(1)}-\bar{E}_{J 2} \sigma_{x}^{(2)}-\chi \sigma_{x}^{(1)} \sigma_{x}^{(2)} .
$$

For instance, when $\bar{E}_{J i}>0, \quad i=1,2$, its four eigenvalues are

$$
\begin{aligned}
& \bar{E}_{J 1}+\bar{E}_{J 2}-\chi, \\
& \bar{E}_{J 1}-\bar{E}_{J 2}+\chi, \\
& \bar{E}_{J 2}-\bar{E}_{J 1}+\chi, \\
& -\bar{E}_{J 1}-\bar{E}_{J 2}-\chi .
\end{aligned}
$$

The corresponding eigenstates are $\left|e_{1}, e_{2}\right\rangle,\left|e_{1}, g_{2}\right\rangle,\left|g_{1}, e_{2}\right\rangle$, and $\left|g_{1}, g_{2}\right\rangle$, where

$$
\begin{aligned}
& \left|e_{i}\right\rangle=\frac{1}{\sqrt{2}}\left(|\uparrow\rangle_{i}-|\downarrow\rangle_{i}\right), \\
& \left|g_{i}\right\rangle=\frac{1}{\sqrt{2}}\left(|\uparrow\rangle_{i}+|\downarrow\rangle_{i}\right) .
\end{aligned}
$$

Because they are also the eigenstates of the two uncoupled charge qubits, when prepared initially at an eigenstate, the system does not evolve to an entangled state even in the presence of interbit coupling. As shown below, one can take advantage of this property to implement the measurement. In addition, this property can be used to construct efficient conditional gates. For instance, if

$$
\bar{E}_{J 1}=\bar{E}_{J 2}=\chi
$$

the controlled-phase-shift (CPS) gate is given by

$$
U_{\mathrm{CPS}}(\tau)=e^{i \chi \tau / \hbar} U
$$

with

$$
U=e^{-i H \tau / \hbar}=\exp \left\{i(\chi \tau / \hbar)\left[\sigma_{x}^{(1)}+\sigma_{x}^{(2)}+\sigma_{x}^{(1)} \sigma_{x}^{(2)}\right]\right\},
$$

at $\tau=\pi \hbar / 4 \chi$. This gate transforms the basis states $\left|e_{1}, e_{2}\right\rangle$, $\left|e_{1}, g_{2}\right\rangle,\left|g_{1}, e_{2}\right\rangle$, and $\left|g_{1}, g_{2}\right\rangle$ as

$$
\left(\begin{array}{c}
\left|e_{1}, e_{2}\right\rangle \\
\left|e_{1}, g_{2}\right\rangle \\
\left|g_{1}, e_{2}\right\rangle \\
\left|g_{1}, g_{2}\right\rangle
\end{array}\right) \rightarrow\left(\begin{array}{cccc}
1 & 0 & 0 & 0 \\
0 & 1 & 0 & 0 \\
0 & 0 & 1 & 0 \\
0 & 0 & 0 & -1
\end{array}\right)\left(\begin{array}{c}
\left|e_{1}, e_{2}\right\rangle \\
\left|e_{1}, g_{2}\right\rangle \\
\left|g_{1}, e_{2}\right\rangle \\
\left|g_{1}, g_{2}\right\rangle
\end{array}\right)
$$

The generation of this conditional two-bit gate is efficient because the condition (24) can be realized in one step via changing the gate voltages $V_{X i}, i=1,2$, and the flux $\Phi_{s}$ simultaneously. Also, the architecture is scalable because multiple charge qubits can be coupled by connecting them in parallel with the large-junction SQUID. If the two Josephson junctions in each Cooper-pair box are replaced by smalljunction dc SQUIDs, any selected pairs of charge qubits (not necessarily neighbors) can be coupled. ${ }^{10}$

\section{MICROWAVE-ASSISTED MACROSCOPIC ENTANGLEMENT}

When a microwave field is applied to the Josephson charge qubit, Rabi oscillations occur in the system. ${ }^{14}$ These oscillations can also be demonstrated by coupling a quantum resonator to the charge qubit. ${ }^{15}$ Here we apply the microwave field to the Cooper-pair box via the gate capacitance, as in Refs. 9 and 14, but each charge qubit is driven by a different microwave field. ${ }^{16}$ In this situation, $n_{X i}$ in Eq. (1) is replaced by

$$
n_{X i}+\hat{n}_{A C i}=n_{X i}+\left(\frac{C_{i} d_{i}}{2 e}\right) \hat{\mathcal{E}}_{A C i} .
$$

Here $d_{i}$ is the thickness of the gate capacitor and

$$
\hat{\mathcal{E}}_{A C i}=\mathcal{E}_{\lambda i} a_{i}+\mathcal{E}_{\lambda i}^{*} a_{i}^{\dagger}
$$

is the microwave electric field in the gate capacitor of the $i$ th Cooper-pair box, where $a_{i}$ is the annihilation operator of the microwave mode. Because the microwave wavelength is much larger than $d_{i}, \mathcal{E}_{\lambda i}$ can be considered constant in the gate capacitor. In the charging regime, the Hamiltonian of the system (including the microwave fields) can be written as

$$
\begin{aligned}
H= & \sum_{i=1}^{2}\left[\varepsilon_{i}\left(V_{X i}\right) \sigma_{z}^{(i)}-\bar{E}_{J i} \sigma_{x}^{(i)}+\hbar \omega_{\lambda i} a_{i} a_{i}^{\dagger}\right. \\
& \left.+\sigma_{z}^{(i)}\left(K_{i} a_{i}+K_{i}^{*} a_{i}^{\dagger}\right)\right]-\chi \sigma_{x}^{(1)} \sigma_{x}^{(2)},
\end{aligned}
$$

where

$$
K_{i}=\left(\frac{E_{c i} C_{i} d_{i}}{2 e}\right) \mathcal{E}_{\lambda i}
$$

Here, we also consider the system working at the degeneracy points $\varepsilon_{i}\left(V_{X i}\right)=0, \quad i=1,2$. When $\hbar \omega_{\lambda i} \approx 2\left|\bar{E}_{J i}\right|$ and under the rotating-wave approximation, the Hamiltonian is cast to 


$$
\begin{aligned}
H= & \sum_{i=1}^{2}\left[-\bar{E}_{J i} \sigma_{x}^{(i)}+\hbar \omega_{\lambda i} a_{i} a_{i}^{\dagger}+\left(K_{i}\left|e_{i}\right\rangle\left\langle g_{i}\right| a_{i}+\text { H.c. }\right)\right] \\
& -\chi \sigma_{x}^{(1)} \sigma_{x}^{(2)} .
\end{aligned}
$$

Without interbit coupling, each Josephson charge qubit exhibits Rabi oscillations between states $\left|e_{i}, l_{i}\right\rangle$ and $\mid g_{i}, l_{i}$ $+1\rangle$, where $\left|l_{i}\right\rangle$ is a photon state with $l_{i}$ photons. For the resonant case with $\hbar \omega_{\lambda i}=2\left|\bar{E}_{J i}\right|$, the eigenvalues of each charge-qubit system are given by

$$
\epsilon_{ \pm}^{(i)}=E_{0 i} \pm \frac{1}{2} \hbar \Omega_{i},
$$

where

$$
E_{0 i}=\hbar \omega_{\lambda i}\left(l_{i}+1\right),
$$

and

$$
\Omega_{i}=\frac{2}{\hbar}\left|K_{i}\right| \sqrt{l_{i}+1}
$$

is the Rabi frequency. Though entanglement occurs between each charge qubit and the nonclassical microwave field, the two qubits do not entangle with each other since the system evolves as

$$
|\Psi(t)\rangle=\left|\psi_{1}(t)\right\rangle\left|\psi_{2}(t)\right\rangle,
$$

where

$$
\left|\psi_{i}(t)\right\rangle=\sin \left(\Omega_{i} t\right)\left|e_{i}, l_{i}\right\rangle+\cos \left(\Omega_{i} t\right)\left|g_{i}, l_{i}+1\right\rangle
$$

if the system is initially prepared at state $\mid g_{1}, g_{2}, l_{1}+1, l_{2}$ $+1\rangle$. However, in the presence of microwave fields, when the interbit coupling is switched on, the coupled-qubit system exhibits complicated quantum oscillations and it will evolve to the entangled state. For instance, in the resonant situation, the eigenvalues are given by

$$
\begin{aligned}
& \varepsilon_{1,4}=E_{01}+E_{02} \pm \hbar \Lambda_{1}, \\
& \varepsilon_{2,3}=E_{01}+E_{02} \pm \hbar \Lambda_{2},
\end{aligned}
$$

where

$$
\Lambda_{1,2}=\left[\left(\Omega_{1} \pm \Omega_{2}\right)^{2}+(\chi / \hbar)^{2}\right]^{1 / 2} .
$$

The state of the coupled-qubit system evolves as

$$
\begin{aligned}
|\Psi(t)\rangle= & C_{1}(t)\left|e_{1}, e_{2}, l_{1}, l_{2}\right\rangle+C_{2}(t)\left|e_{1}, g_{2}, l_{1}, l_{2}+1\right\rangle \\
& +C_{3}(t)\left|g_{1}, e_{2}, l_{1}+1, l_{2}\right\rangle \\
& +C_{4}(t)\left|g_{1}, g_{2}, l_{1}+1, l_{2}+1\right\rangle .
\end{aligned}
$$

For the system prepared initially at $\left|g_{1}, g_{2}, l_{1}+1, l_{2}+1\right\rangle$,

$$
\begin{aligned}
& C_{1}(t)=\frac{1}{2}\left\{R_{2}(t)-R_{1}(t)+i(\chi / \hbar)\left[S_{2}(t)-S_{1}(t)\right]\right\}, \\
& C_{2}(t)=\frac{1}{2}\left[\left(\Omega_{1}+\Omega_{2}\right) S_{1}(t)+\left(\Omega_{1}-\Omega_{2}\right) S_{2}(t)\right],
\end{aligned}
$$

$$
\begin{aligned}
& \left.C_{3}(t)=\frac{1}{2}\left[\left(\Omega_{1}+\Omega_{2}\right) S_{1}(t)-\left(\Omega_{1}-\Omega_{2}\right) S_{2} t\right)\right], \\
& C_{4}(t)=\frac{1}{2}\left\{R_{1}(t)+R_{2}(t)+i(\chi / \hbar)\left[S_{1}(t)+S_{2}(t)\right]\right\},
\end{aligned}
$$

where

$$
R_{i}(t)=\cos \left(\Lambda_{i} t\right), \quad S_{i}(t)=\frac{\sin \left(\Lambda_{i} t\right)}{\Lambda_{i}} .
$$

For a two-level system interacting with a single-mode field, the Rabi oscillations can be explained using either quantum or semiclassical theory, where the single-mode field is described quantum mechanically or treated as a classical field. ${ }^{17}$ Here the quantum oscillations of coupled charge qubits (namely, the Rabi oscillations in coupled two-level systems) are studied using quantum theory, where the microwave field coupled to each qubit is quantized. This also applies to the classical-field case, in which the quantum oscillations are still described by Eq. (40), but $\left|e_{1}, e_{2}, l_{1}, l_{2}\right\rangle$, $\left|e_{1}, g_{2}, l_{1}, l_{2}+1\right\rangle, \quad\left|g_{1}, e_{2}, l_{1}+1, l_{2}\right\rangle, \quad$ and $\mid g_{1}, g_{2}, l_{1}+1, l_{2}$ $+1\rangle$ are replaced by $\left|e_{1}, e_{2}\right\rangle,\left|e_{1}, g_{2}\right\rangle,\left|g_{1}, e_{2}\right\rangle$, and $\left|g_{1}, g_{2}\right\rangle$.

Figure 3 shows the occupation probability $\left|C_{1}(t)\right|^{2}$ as a function of time $t$. For instance, when $\left|C_{1}(t)\right|^{2} \simeq 1$, both charge qubits are in their excited states. It can be seen that $\left|C_{1}(t)\right|^{2}$ looks very different when the interbit coupling is switched on or off. The macroscopic entanglement between the two coupled qubits can be explicitly shown at $\Omega_{1}$ $=\Omega_{2}(=\Omega)$. In this case, when $t_{\mathrm{ent}}=n \pi \hbar / W \chi$, with $n$ $=1,2,3, \ldots$, and

$$
W=\left[(2 \hbar \Omega / \chi)^{2}+1\right]^{1 / 2} .
$$

$|\Psi(t)\rangle$ becomes

$$
\begin{aligned}
\left|\Psi\left(t_{\mathrm{ent}}\right)\right\rangle= & C_{1}\left(t_{\mathrm{ent}}\right)\left|e_{1}, e_{2}, l_{1}, l_{2}\right\rangle \\
& +C_{4}\left(t_{\mathrm{ent}}\right)\left|g_{1}, g_{2}, l_{1}+1, l_{2}+1\right\rangle,
\end{aligned}
$$

where

$$
\begin{gathered}
C_{1}\left(t_{\mathrm{ent}}\right)=\frac{1}{2}[-\cos (n \pi)+\exp (\text { in } \pi / W)], \\
C_{4}\left(t_{\mathrm{ent}}\right)=\frac{1}{2}[\cos (n \pi)+\exp (\text { in } \pi / W)] .
\end{gathered}
$$

The peaks away from either zero or 1 shown in Fig. 3(a) correspond to this kind of entangled state. Furthermore, if suitable values of $W$ are taken, the maximally entangled state with $\left|C_{1}\right|^{2}=\left|C_{4}\right|^{2}=\frac{1}{2}$ can be derived. This state is a macroscopic Schrödinger-cat state of the two charge qubits. For instance, if $\hbar \Omega / \chi=\sqrt{3} / 2$, the coupled-qubit system evolves to the maximally entangled state at the times given by

$$
t_{\mathrm{ent}}^{(\max )}=(2 l+1) \pi \hbar / 2 \chi, \quad l=0,1,2, \ldots .
$$

This entangled state corresponds to the half-probability peaks in Fig. 3(c). 

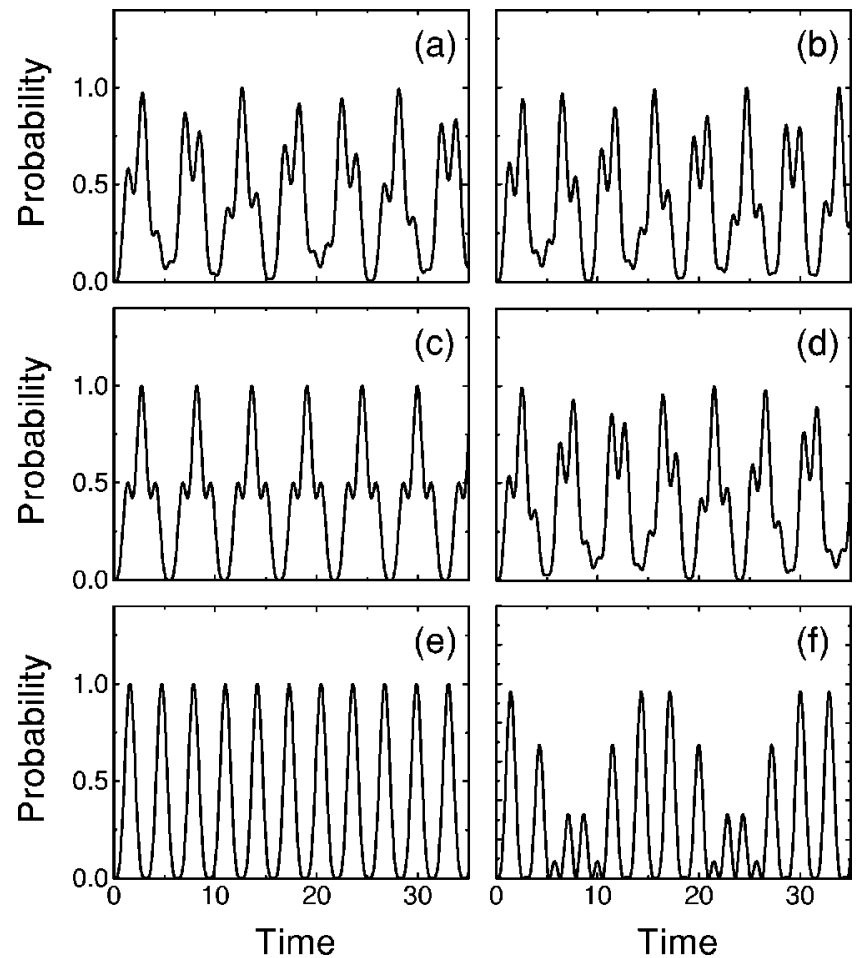

FIG. 3. Occupation probability $\left|C_{1}(t)\right|^{2}$ as a function of time. (a) $\Omega_{2}=\Omega_{1}, \chi / \hbar=\Omega_{1} ; \quad$ (b) $\Omega_{2}=1.2 \Omega_{1}, \chi / \hbar=\Omega_{1} ; \quad$ (c) $\Omega_{2}$ $=\Omega_{1}, \chi / \hbar=\sqrt{3} \Omega_{1} / 2 ; \quad$ (d) $\Omega_{2}=1.2 \Omega_{1}, \chi / \hbar=\sqrt{3} \Omega_{1} / 2$; (e) $\Omega_{2}$ $=\Omega_{1}, \chi=0$; (f) $\Omega_{2}=1.2 \Omega_{1}, \chi=0$. The time is in units of $\Omega_{1}^{-1}$.

\section{QUANTUM MEASUREMENT}

To implement a readout, we bias a current pulse $I_{b}$ to the qubit circuit (see Fig. 2), as in the single-qubit case. ${ }^{9}$ Now, a term $-\Phi_{0} I_{b} \hat{\delta} / 2 \pi$, with

$$
\hat{\delta}=\frac{1}{4}\left[\hat{\gamma}_{L}+\hat{\gamma}_{R}+\sum_{i=1,2}\left(\hat{\phi}_{i A}-\hat{\phi}_{i B}\right)\right],
$$

should be added to the Hamiltonian (1), where $\hat{\delta}$ is the average phase drop of the total qubit circuit and it can be written as

$$
\hat{\delta}=\hat{\gamma}-\frac{\pi \Phi_{s}}{2 \Phi_{0}}
$$

with $\hat{\gamma}=\frac{1}{2}\left(\hat{\gamma}_{L}+\hat{\gamma}_{R}\right)$. Here we set the flux $\Phi_{s}$ equal to zero to have a larger effective Josephson coupling energy. In the spin- $\frac{1}{2}$ representation based on charge states, the Hamiltonian of the system is also reduced to Eq. (12). The interbit coupling is here induced by the bias current and given by

$$
\chi=L_{J} I_{c 1} I_{c 2} \sin ^{2}\left(\gamma_{0} / 2\right),
$$

where the effective inductance is

$$
L_{J}=\frac{\Phi_{0}}{2 \pi I_{0} \cos \gamma_{0}},
$$

and

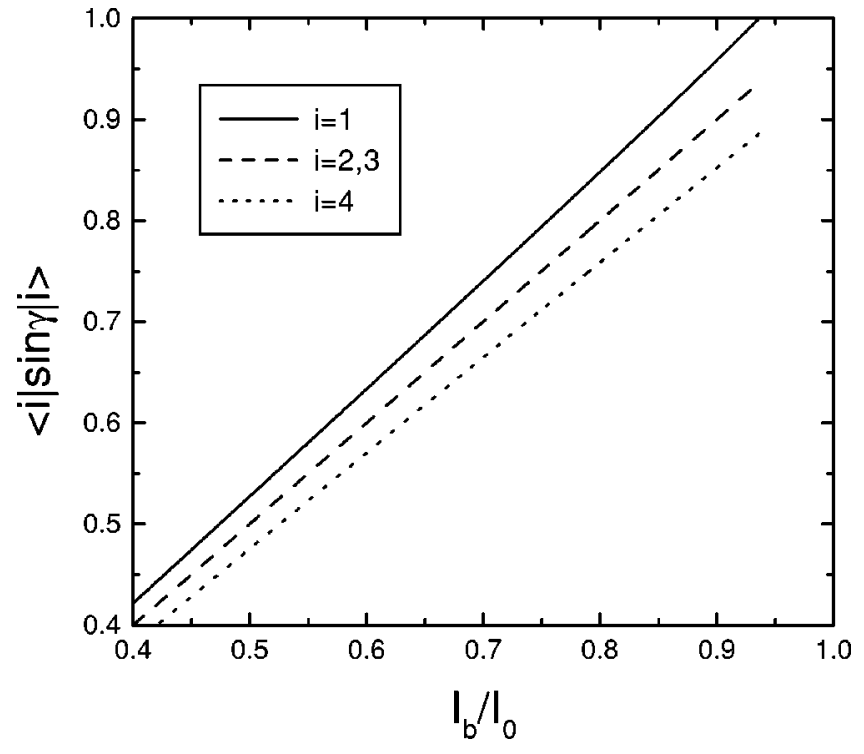

FIG. 4. Eigenstate dependence of the supercurrent through the SQUID as a function of the bias current $I_{b}$. Here, $E_{J 1}=E_{J 2}$ $=\frac{1}{5} E_{J 0}^{s}, \quad|1\rangle=\left|e_{1}, e_{2}\right\rangle, \quad|2\rangle=\left|e_{1}, g_{2}\right\rangle, \quad|3\rangle=\left|g_{1}, e_{2}\right\rangle, \quad$ and $|4\rangle$ $=\left|g_{1}, g_{2}\right\rangle$.

$$
\gamma_{0}=\sin ^{-1}\left(I_{b} / I_{0}\right),
$$

with $I_{0}=4 \pi E_{J 0}^{s} / \Phi_{0}$, and $I_{b}<I_{0}$. The intrabit couplings are

$$
\bar{E}_{J i}=E_{J i} \cos \left(\gamma_{0} / 2\right) \xi_{i}
$$

where

$$
\xi_{i}=1-\alpha\left(\eta_{i}^{2}+3 \eta_{j}^{2}\right) \sin ^{2}\left(\gamma_{0} / 2\right),
$$

with

$$
\alpha=\frac{2+\cos \gamma_{0}}{8 \cos ^{3} \gamma_{0}},
$$

and $i, j=1,2(i \neq j)$. The supercurrent through the SQUID,

$$
\begin{aligned}
I_{0} \sin \hat{\gamma}= & I_{b}-\sin \left(\gamma_{0} / 2\right)\left[I_{c 1} \sigma_{X}^{(1)}+I_{c 2} \sigma_{X}^{(2)}\right] \\
& +\frac{1}{4 I_{0}} \tan \gamma_{0}\left[I_{c 1}^{2}+I_{c 2}^{2}+2 I_{c 1} I_{c 2} \sigma_{X}^{(1)} \sigma_{X}^{(2)}\right],
\end{aligned}
$$

has contributions from both the bias current and the current from the Josephson charge qubits.

At the working points with $\varepsilon_{i}\left(V_{X i}\right)=0$, the eigenstates of the system are also $\left|e_{1}, e_{2}\right\rangle,\left|e_{1}, g_{2}\right\rangle,\left|g_{1}, e_{2}\right\rangle$, and $\left|g_{1}, g_{2}\right\rangle$. In Fig. 4, we show the dependence of the supercurrents through the SQUID on the eigenstates of the charge-qubit system. The supercurrents through the SQUID increase with the bias current and the difference between the supercurrents at different (nondegenerate) eigenstates widens. For the measurement setup shown in Fig. 2, the supercurrent through the SQUID is the largest at the eigenstate $\left|e_{1}, e_{2}\right\rangle$ and it first reaches the maximal value $I_{0}$ (namely, the critical current) when the bias current $I_{b}$ approaches a value $I_{S W}$ near $I_{0}$. 
Around this value, the supercurrent through the SQUID switches, with a very large probability $P_{1}$, from the zerovoltage state to the dissipative nonzero-voltage state in the quasiparticle-current branch and the measurement on the voltage is carried out. However, due to environmental noise as well as thermal and quantum fluctuations, the switching actually occurs before the supercurrent through the SQUID reaches $I_{0}$. At $I_{b} \sim I_{S W}$, the supercurrents through the SQUID will also switch to the nonzero voltage state at other eigenstates, but the switching probabilities are small. In the ideal case, if the difference between the large switching probability $P_{1}$ and the small ones is close to 1 , then, in principle, a single-shot readout would be achieveable. As shown in Ref. 9, the Josephson-junction switching experiment can provide sufficient accuracy to discriminate the state $\left|e_{1}, e_{2}\right\rangle$ from others.

The operation and readout of the macroscopic entanglement of the coupled-qubit system can be implemented by simultaneously applying a pulsed microwave field (with the same duration $\tau$ ) to each charge qubit. The sequence would be:

(i) before the microwave fields are applied, the flux $\Phi_{s}$ through the SQUID is set equal to zero and no interbit coupling exists;

(ii) the flux $\Phi_{s}$ is switched on to a certain nonzero value exactly at the start of the microwave pulse and off at the end of the microwave pulse. Within the microwave pulse duration $\tau$, the evolution of the system is described by Eq. (40);

(iii) a pulsed bias current $I_{b}$ is applied to perform a measurement after the microwave pulse.

During the measurement, the quantum state of the chargequbit system collapses to the eigenstate $\left|e_{1}, e_{2}\right\rangle$ with probability $\left|C_{1}(\tau)\right|^{2}$. This probability is proportional to the switching probability $P_{1}$ of the SQUID. Because of relaxation, the envelope of the measured switching probability $P_{1}$ decays exponentially with time. This is used to obtain the relaxation time. ${ }^{2,9}$ Ramsey fringes of the probability $P_{1}$ can be used ${ }^{9}$ to determine the decoherence time of the coupledqubit system. For each given microwave pulse duration $\tau$, through repeated measurements, one can determine the occupation probability $\left|C_{1}(\tau)\right|^{2}$ and thus deduce the information about the macroscopic entanglement between the coupled charge qubits [see Figs. 3(a) and 3(c)].

\section{DISCUSSION AND CONCLUSION}

Finally, we estimate some important parameters using available quantities for the single charge qubit. Here we consider the maximally entangled case shown in Fig. 3(c), in which $\Omega_{1}=\Omega_{2}=\Omega$, and

$$
\frac{\chi}{\hbar}=\frac{\sqrt{3}}{2} \Omega .
$$

Taking $2 \pi / \Omega \simeq 0.22 \mu \mathrm{s}$, as derived from the Rabi oscillation of the measured switching probability, ${ }^{9}$ we have $\chi / \hbar$ $\approx 0.25 \mathrm{GHz}$. Reference 9 also gives $E_{J} / \hbar \approx 16.5 \mathrm{GHz}$. Choosing

$$
E_{J 0}^{s} \approx 5 E_{J i} \approx 5 E_{J},
$$

and using the relation for $\chi$, we obtain $\Phi_{s} \approx 0.35 \Phi_{0}$. For $\Phi_{s}=0$, the expansion parameters are

$$
\eta_{i}=\frac{I_{0 i}}{I_{0}} \approx 0.05
$$

for $E_{J 0}^{s} \approx 5 E_{J i}$. When $\Phi_{s} \approx 0.35 \Phi_{0}$, they become $\eta_{i} \approx 0.14$. The results are sufficiently accurate when $\bar{E}_{J i}$ and $\chi$ are retained up to second- and higher-order terms in the expansion parameters $\eta_{i}$. When $\Phi_{s}$ approaches $\Phi_{0} / 2$, the interbit coupling strengthens. The reduced Hamiltonian of the system also has the same form as Eq. (12), but higher-order terms in the expansion parameters should be included to obtain accurate results.

Here we consider the charging regime with $E_{c i} \gg E_{J i}$ in order to obtain analytical results. We expect that the interbit coupling can still be realized in the regime with $E_{c i} \sim E_{J i}$, i.e., the regime used by the Saclay group in the experiment on a single Josephson qubit. ${ }^{9}$ In this latter regime, the results can only be obtained numerically, but a relatively long decoherence time would be expected for the coupled-qubit system to work at the degeneracy points because at these points the states are more stable against the variations of both the offset charges and the flux $\Phi_{e}$ or $\Phi_{s}$.

Very recently, quantum oscillations were experimentally observed in two coupled charge qubits. ${ }^{18}$ Also, a novel method for the controllable coupling of charge qubits was proposed using a variable electrostatic transformer. ${ }^{19}$ In contrast with our interbit coupling scheme, these studies involve capacitively-coupled (as opposed to inductively-coupled) charge qubits. The main advantage of this inductive coupling among qubits is that it allows a controllable link between any selected qubits, not necessarily nearest neighbors.

In conclusion, we employ a large-junction SQUID to couple Josephson charge qubits and implement a readout. This architecture is readily scalable to multiple qubits. When the system works at the degeneracy points, where the dephasing effects are suppressed, it is shown that the macroscopic entanglement can be generated with the assistance of microwave fields. Also, we show the quantum measurement of the macroscopic entanglement.

\section{ACKNOWLEDGMENTS}

We thank X. Hu and B. Plourde for useful comments. We acknowledge support from the U.S. ARDA, AFOSR, and the U.S. National Science Foundation Grant No. EIA-0130383.

398, 786 (1999).

${ }^{3}$ Y. Makhlin, G. Schön, and A. Shnirman, Nature (London) 398, 305 (1999).

${ }^{4}$ J. E. Mooij, T. P. Orlando, L. Levitov, L. Tian, C. H. van der Wal,

\footnotetext{
*Corresponding author. Email address: nori@umich.edu

${ }^{1}$ See, e.g., Y. Makhlin, G. Schön, and A. Shnirman, Rev. Mod. Phys. 73, 357 (2001), and references therein.

${ }^{2}$ Y. Nakamura, Yu. A. Pashkin, and J. S. Tsai, Nature (London)
} 
and S. Lloyd, Science 285, 1036 (1999); T. P. Orlando, J. E. Mooij, L. Tian, C. H. van der Wal, L. S. Levitov, S. Lloyd, and J. J. Mazo, Phys. Rev. B 60, 15398 (1999).

${ }^{5}$ C. H. van der Wal, A. C. J. ter Haar, F. K. Wilhelm, R. N. Schouten, C. J. P. M. Harmans, T. P. Orlando, S. Lloyd, and J. E. Mooij, Science 290, 773 (2000).

${ }^{6}$ J. R. Friedman, V. Patel, W. Chen, S. K. Tolpygo, and J. E. Lukens, Nature (London) 406, 43 (2000).

${ }^{7}$ Y. Yu, S. Han, X. Chu, S.-I. Chu, and Z. Wang, Science 296, 889 (2002)

${ }^{8}$ J. M. Martinis, S. Nam, J. Aumentado, and C. Urbina, Phys. Rev. Lett. 89, 117901 (2002).

${ }^{9}$ D. Vion, A. Aassime, A. Cottet, P. Joyez, H. Pothier, C. Urbina, D. Esteve, and M. H. Devoret, Science 296, 886 (2002); A. Cottet, D. Vion, A. Aassime, P. Joyez, D. Esteve, and M. H. Devoret, Physica C 367, 197 (2002).

${ }^{10}$ J. Q. You, J. S. Tsai, and F. Nori, Phys. Rev. Lett. 89, 197902 (2002). A longer version of this work is available online in cond-mat/0306208.

${ }^{11}$ F. Plastina, R. Fazio, and G. M. Palma, Phys. Rev. B 64, 113306 (2001)

${ }^{12}$ J. Siewert and R. Fazio, Phys. Rev. Lett. 87, 257905 (2001).
${ }^{13}$ A. Blais, A. Maassen van den Brink, and A. M. Zagoskin, Phys. Rev. Lett. 90, 127901 (2003).

${ }^{14}$ Y. Nakamura, Yu. A. Pashkin, and J. S. Tsai, Phys. Rev. Lett. 88, 047901 (2002)

${ }^{15}$ A. D. Armour, M. P. Blencowe, and K. C. Schwab, Phys. Rev. Lett. 88, 148301 (2002); O. Buisson and F. W. J. Hekking, in Macroscopic Quantum Coherence and Quantum Computing (Kluwer Academic, Dordrecht, 2001), pp. 137-145; F. W. J. Hekking, O. Buisson, F. Balestro, and M. G. Vergniory, cond-mat/0201284 (unpublished); F. Marquardt and C. Bruder, Phys. Rev. B 63, 054514 (2001).

${ }^{16}$ J. Q. You and F. Nori, cond-mat/0306207, Phys. Rev. B (to be published) studied the quantum dynamics of a Cooper-pair box with a nonclassical microwave magnetic field going through the superconducting loop, instead of a microwave voltage (electric) oscillation applied to the gate capacitance.

${ }^{17}$ M. O. Scully and M. S. Zubairy, Quantum Optics (Cambridge University, Cambridge, England, 1997), Chaps. 5 and 6.

${ }^{18}$ Yu. A. Pashkin, T. Yamamoto, O. Astafiev, Y. Nakamura, D. V. Averin, and J. S. Tsai, Nature (London) 421, 823 (2003).

${ }^{19}$ D. V. Averin and C. Bruder, cond-mat/0304166 (unpublished). 